Cuadernos de Historia Contemporánea

ISSN: 0214-400X

\title{
Los Historical Game Studies como línea de investigación emergente en las Humanidades
}

Federico Peñate Domínguez ${ }^{1}$

Adam Chapman: Digital Games as History. How Videogames Represent the Past and Offer Access to Historical Practice. Oxon/New York, Routledge, 2016. 290 pp.

Andrew Elliott y Matthew Wilhelm Kapell (eds.): Playing with the Past. Digital Games and the Simulation of History. London/New York, Bloomsbury, 2013. 388 pp.

William Uricchio: "Simulation, history, and computer games", en Joost Raessens y Jeffrey Goldstein: Handbook of Computer Game Studies. Cambridge (Mass.): The MIT Press, 2005. 327-338 pp.

Tobias Winnerling y Florian Kerschbaumer (eds.): Early Modernity and Video Games. Newcastle upon Thyne, Cambridge Scholars Publishing, 2014. 265 pp.

"La historia es nuestro patio de juego": Esta frase lapidaria se ha convertido en el mantra de la popular serie de videojuegos Assassin's Creed. Producida y desarrollada por la franco-canadiense UbiSoft, una de las empresas multinacionales más potentes de la industria actual, la franquicia nos pone en la piel de agentes históricos y nos sitúa en distintos contextos del pasado que somos capaces de recorrer y en cuyos acontecimientos podemos participar. Los periodos representados incluyen la Tercera Cruzada, la Italia Renacentista, la Guerra de Independencia Estadounidense, el Caribe durante la Edad de Oro de la Piratería y la Revolución Francesa, por citar algunos ejemplos. Entre los factores de su éxito destacan sus detalladas réplicas de la vestimenta y los artefactos característicos de cada época, la aparición de personajes históricos de gran relevancia y, por encima de todo ello, las minuciosas reproducciones de ciudades históricas como San Juan de Acre, Florencia o París. El detalle con el que se han plasmado sus calles y edificios, en especial aquellas construcciones monumentales mundialmente reconocidas, es uno de los factores que explican que la franquicia haya vendido más de 77 millones de copias en sus casi diez años de vida ${ }^{2}$. Diez años que han presenciado la salida al

1 FPU15/00414 / Proyecto de investigación: HAR2015-64155P Universidad Complutense de Madrid (España) E-mail:federico.penate.dominguez@gmail.com.

2 Ubisoft Sells 77 Million Units Of The Games Assassin's Creed. N2G.us, www.n2g.us/2014/10/ubisoft-sells-77million-units-of-games.html\#.WJXdDhvhC00 (Consultado 04/02/2017). 
mercado de los nueve grandes títulos principales de la saga, a los que se le suman once más de carácter secundario.

El de Assassin's Creed no es un caso aislado, ni mucho menos. Desde los albores del juego digital, el pasado de la humanidad ha servido de escenario sobre el cual edificar los mundos ludoficcionales ${ }^{3}$ de estos productos de entretenimiento. Es más, la tendencia a utilizar el pasado de la Humanidad como telón de fondo, en boga desde hace más de tres décadas, aún no se ha agotado. Así lo demuestran los datos referentes al lanzamiento de nuevos videojuegos. Sólo en 2016, cinco de los treinta productos más populares trataban explícitamente acontecimientos y procesos del pasado o hacían referencia a ellos ${ }^{4}$. El éxito de estas manifestaciones culturales y de entretenimiento digital no ha pasado desapercibido a los historiadores (mayoritariamente anglofonos), lo que ha generado un notable volumen de bibliografía y estudios cuyos orígenes se remontan a hace ya más de una década. En lo que respecta a la franquicia citada en la introducción, autoras como Adrienne Shaw ${ }^{5}$, Douglas N. Dow ${ }^{6}$ y Emil Ludendal Hammar ${ }^{7}$ se han aproximado a su estudio desde distintas perspectivas como la teoría del género, la historia del arte y los estudios postcoloniales, todo ello sin abandonar la disciplina de la Historia. Los resultados de sus trabajos son imprescindibles a la hora de comprender los discursos sobre el pasado que esta saga de videojuegos enuncia, además de elementos, tales como metanarrativas históricas y tendencias culturales, que han influido en la construcción de dichos relatos.

A pesar de ello, sólo en el último lustro el estudio de las simulaciones digitales del pasado ha empezado a adquirir una naturaleza disciplinar y un carácter metódico. Los primeros trabajos raramente superaban el mero análisis de contenido, lo que permitía únicamente arañar la superficie del objeto de estudio e impedía comprender no sólo el tratamiento que este novedoso medio le da a la historia, sino también su potencial para generar nuevos modos de escribir sobre el pasado, alternativos a las formas tradicionales y hegemónicas. Sin embargo, con el tiempo han ido aumentado las aportaciones teórico-metodológicas procedentes de las Humanidades: categorías extraídas de la Filosofía, la Historia y la Antropología, por citar unos ejemplos, se han incorporado al corpus conceptual. Esta progresiva adaptación se ve especialmente bien reflejada en la revista Rethinking History. The Journal of Theory and Practice. Desde 2007, la publicación ha ido dando progresiva cabida a estudios sobre la Historia en formato lúdico, llegando en 2016 a publicar un número especial, Challenge the Past, dedicado exclusivamente a esta tendencia. En su introducción se presentan los historical game studies como una nueva disciplina prácticamente consolidada, se hace un repaso a su pasado y se realizan una serie de recomendaciones,

3 Planells de la Maza, Antonio J.: Videojuegos y mundos de ficción. De Super Mario a Portal, Madrid, Cátedra: Signo e Imagen, 2015.

42016 in video gaming. Wikipedia.org https://en.wikipedia.org/wiki/2016_in_video_gaming (Consultado 04/02/2016).

5 Shaw, Adrienne: “The Tyranny of Realism: Historical accuracy and politics of representation in Assassin's Creed III ”, Loading... Journal of the Canadian Game Studies Organization, 9-14 (2015), pp. 4-24.

6 Dow, Douglas N.: "Historical Veneers. Anachronism, Simulation, and Art History in Assassin's Creed II", en Elliott, Andrew B. R. y Kapell, Matthew Wilhelm (eds.): Playing with the Past. Digital Games and the Simulation of History, London/New York, Bloomsbury, 2013, pp. 215-232.

7 Hammar, Emil Ludendal: "Counter-hegemonic commemorative play: marginalized pasts and the politics of memory in the digital game Assassin's Creed: Freedom Cry", Rethinking History. The Journal of Theory and Practice, special issue Challenge the Past (2016), pp. 1-24. 
fundamentadas en el estatus que sus autores ostentan dentro de la especialidad, sobre la dirección que deberían tomar los futuros estudios en la materia. En la actualidad, la necesidad más acuciante recae en el perfeccionamiento del andamiaje intelectual sobre el que la disciplina ha de sostenerse. En palabras de los autores:

The importance of pursuing the creation of an analytical language to describe the ways in which digital technologies continue to change the ways we engage with the past, particularly given that these technological changes tend to continually outpace our efforts to understand them ${ }^{8}$.

Sin embargo, como bien refleja dicha introducción, ya se ha recorrido una buena parte del camino inicial. El propósito de la presente nota bibliográfica es dar a conocer a la comunidad académica hispanohablante las obras fundamentales sobre las cuales la disciplina de los historical game studies ha sido edificada, así como ofrecer una breve reseña del contenido de las mismas y subrayar sus aportaciones más interesantes.

A pesar de que existen obras anteriores que tratan sobre la adaptación de discursos históricos a formatos lúdicos (y con ello no nos referimos únicamente a los de carácter digital, pues el estudio de juegos de tablero, simulaciones con miniaturas y wargames ambientados en el pasado no es una novedad), Chapman, Foka y Westin consideran a William Uricchio el padre de los historical game studies (un padre ausente, pues el autor ha enfocado después su investigación a otros campos del conocimiento). Esta opinión la comparten la amplia mayoría de sus colegas, a juzgar por la naturaleza recurrente de las citas al que se considera el trabajo fundacional de la disciplina. Se trata de "Simulation, history, and computer games", un capítulo incluido en la obra colectiva Handbook of Computer Game Studies que data del año 2005, momento en el que la estructura de los game studies terminaba de cuajar como resultado de los arduos debates entre narratólogos y ludólogos 9 . En este escueto apartado de 12 páginas, sin embargo, se evidencian los primeros pasos de lo que se ha acabado convirtiendo en una línea de investigación madura. Existe consenso al afirmar que Uricchio, mediante dicho trabajo, estableció la hoja de ruta de los historical game studies y sus principales ejes de estudio.

En primer lugar, el autor identificó el principal motivo por el que los historiadores habían desatendido el medio del ocio digital: la interactividad, esto es, la capacidad del lector (en este caso, jugador) de intervenir activamente en el discurso histórico que se le presenta y modificarlo parcialmente:

But if we take interactivity to be one of the distinguishing characteristics of computer games, the interaction between a present-day player and the representation of a historically specific world would seem to challenge any notion of a unique configuration of historical 'fact' and 'fixity', giving away instead to the historically inconsistent and ludic. Such an interaction provokes fundamental questions regarding the place of computer games in systems of historical representation... ${ }^{10}$.

8 Chapman, Adam; Foka, Anna y Westin, Jonathan: "Introduction: what is historical game studies?", Rethinking History. The Journal of Theory and Practice, special issue Challenge the Past (2016) p. 10.

9 Planells de la Maza, Antonio J.: Videojuegos y mundos de ficción. De Super Mario a Portal, Madrid, Cátedra: Signo e Imagen, 2015, pp. 75-83.

10 Uricchio, William: "Simulation, history, and computer games", en Raessens, Joost y Goldstein, Jeffrey: Handbook of Computer Game Studies, Cambridge (Mass.): The MIT Press, 2005, p. 327. 
Frente a esta aparente contradicción irreconciliable entre una concepción de la historia fija, inmutable y característica de narrativas más tradicionales (con la posible excepción, quizá, de la virtual history de la que hablaba Niall Ferguson), y una historia mutable e interactiva, Uricchio ofreció una solución que sería explorada en profundidad por autores posteriores. En las conclusiones de su capítulo considera que la esencia del discurso histórico, su base teórica, presente en un videojuego debe manifestarse en el sistema de reglas, pues es el aparato que gobierna la ludo-narrativa y, por tanto, el relato histórico a construir entre el desarrollador del videojuego y el consumidor.

Por otro lado, Uricchio realizó una primera clasificación de las formas en las que la historia se manifiesta en los productos de entretenimiento digital. En su opinión, existen dos tipos de videojuegos históricos. El primer grupo abarca aquellos que hacen referencia a un evento histórico particular: éstos tienden a maximizar el detalle y la precisión en sus representaciones (pone como ejemplo el título Grand Prix Legends, inspirado en las carreras de bólidos de los años sesenta del siglo XX, en su esfuerzo por representar los coches de la época de la forma más exacta posible) a costa de limitar las acciones y la libertad del jugador, quien normalmente posee la perspectiva de un agente histórico. Este tipo de productos construyen sus mundos ludo-ficcionales de carácter histórico "...through encrusted layers of historical scholarship, training, and popular memory" "11. De forma completamente opuesta, la segunda categoría la componen juegos que tratan de representar procesos históricos de largo recorrido de una forma abstracta y estructuralista, en los que el usuario trasciende las limitaciones del sujeto histórico y ocupa una posición superior, cuasi divina $^{12}$, a través de la cual toma decisiones estratégicas y es capaz de percibir sus consecuencias a largo plazo (puede establecerse una relación con la larga duración braudeliana). A diferencia de las simulaciones específicas:

These less specifically situated games tend to be more evidently structured by unspoken historical principle (or better, ideology), rendering them closer to structuralist notions of history ${ }^{13}$.

Esta diferenciación entre simulaciones específicas y no específicas se ha convertido en los cimientos sobre los que se ha desarrollado el estudio epistemológico del videojuego de corte histórico, como veremos con posterioridad.

Quizá lo más notable del trabajo de Uricchio sea que añade las formas lúdicas de escribir historia a la corriente de pensamiento postestructuralista. La naturaleza del videojuego, alejada de las concepciones de la historia académica que normalmente se consideran a sí mismas científicas y objetivas, permite poner en primer plano las características de la historia entendida como relato construido por los historiadores $\mathrm{y}$, en general, por la sociedad. Estas son, fundamentalmente, su parcialidad idiosin-

11 Uricchio, William: "Simulation, history..."., p. 328.

12 Gerard A. Vorhees ha realizado una interesante aportación teorética en relación a la posición del jugador en este tipo de productos, centrados por lo general en la estrategia y la gestión. En contraste a la concepción general del jugador como sujeto divino - causada, en parte, por la generalización del término "god-like games"-, el autor señala que el rol del usuario comparte una serie de características fundamentales con el sujeto cartesiano. Vorhees, Gerard A.: "I Play Therefore I Am. Sid Meier's Civilization, Turn-Based Strategy Games, and the Cogito", Games and Culture, 4-3 (2009), pp. 254-275.

13 Uricchio, William: "Simulation, history..."., p. 328. 
crática, su tendencia a 'deformar' y delimitar el pasado - que ya se ha perdido, y del cual sólo quedan vestigios a interpretar $-\mathrm{y}$ su inevitable condición presentista. La historia en formato lúdico subraya estas características, poniendo a disposición del usuario una serie de herramientas que, aunque de forma limitada, le permiten alterar a placer el relato sobre el pasado:

Games by definition subvert the project of consolidation and certainly associated with the former brand of history. Instead, predicated as they are upon a reflexive awareness of the construction of history, they seem relevant to the notion of history as a time-bound meaning situated in an ever-changing present ${ }^{14}$.

Paralelamente, el autor detecta una contradicción entre el potencial subversivo del medio y las tendencias seguidas por los desarrolladores de videojuegos a la hora de crear un producto cuyo mundo virtual esté situado en el pasado, con las que adopta una actitud crítica. Por regla general, los creativos tienden a adherirse a interpretaciones de la historia hegemónicas y estructuralistas, estando la mayor parte de sus esfuerzos dedicados a lograr el máximo detalle histórico y asumiendo, de forma acrítica, ciertas meta-narrativas (lo que incluye explicaciones masculinas, progresistas y eurocéntricas, por citar algunos de sus componentes). En los últimos párrafos, Uricchio realiza el siguiente llamamiento tanto a historiadores como desarrolladores:

By embedding various historiographic epistemologies as structuring agencies rather than relying implicitly on narratives of truth, progress, and the American way, a new dimension could be added to play, more coherently addressing history's rich complexity and relevance ${ }^{15}$.

La publicación del trabajo de Uricchio trajo consigo un incremento en las publicaciones sobre videojuegos de corte histórico, aunque la mayoría han aparecido en revistas especializadas en disciplinas distintas de la historia. Por ello, la publicación en 2013 de Playing with the Past, una obra colectiva centrada en el estudio de las simulaciones digitales del pasado, ha sido considerada como el segundo hito en la trayectoria de los historical game studies. Sus 23 capítulos, organizados en cinco bloques temáticos, son el resultado del trabajo colaborativo de 29 investigadores. La variedad y el número de académicos implicados en su elaboración se han visto reflejados en las nuevas aportaciones teóricas que la obra ha introducido. Así, cada una de las secciones gira en torno a una problemática distinta, arrojando nueva luz sobre cuestiones clave no sólo del videojuego como formato para darle sentido al pasado, sino de la disciplina histórica en general.

El primer bloque aborda la cuestión de la causalidad, la contingencia y la teleología. En este sentido, el formato lúdico sacude los cimientos de la última, pues el final del relato no está escrito, sino que queda a discreción del jugador. Asimismo, la causalidad es percibida de forma distinta, fruto de las acciones propias realizadas durante el acto de juego, y de naturaleza repetitiva, en lugar de relegadas a la condición de únicas y pretéritas, como las transmiten los formatos tradicionales. Finalmente, Chapman introduce el concepto gibsoniano de affordances como modo de entender

14 Uricchio, William: "Simulation, history..."., p. 333.

15 Uricchio, William: “Simulation, history...,., p. 336. 
la contingencia. Esta perspectiva ecológica, que el autor desarrolla en trabajos posteriores, hace énfasis en la relación recíproca de los grupos humanos con el medio, lo que les permite realizar una serie de acciones. Según el autor, los videojuegos son un medio particularmente privilegiado a la hora de transmitir esta idea. La segunda sección se centra en una de las críticas realizadas por Uricchio a la industria videolúdica: su particular atracción por las narrativas triunfalistas generadas en Occidente y que justifican el dominio de las potencias de herencia grecorromana y judeocristiana. Los estudios incluyen análisis sobre la figura del bárbaro en juegos ambientados en la Antigüedad Clásica, en el concepto de indígena y su trato marginal en el contexto de la colonización de América, y los discursos en torno al concepto dela dominancia en el enfrentamiento entre civilizaciones europeas y no europeas, entre otros.

Seguidamente, la cuestión de la construcción artificial del discurso histórico figura como el eje central del tercer apartado del libro. La edificación de un relato y, por tanto, la dotación de un significado concreto al pasado no es exclusiva del desarrollador de la obra digital: el usuario, mediante la participación activa, también es responsable. Algunos de los capítulos de la sección van más allá y estudian la práctica conocida como modding, esto es, la alteración del código de un videojuego con el fin de modificar sus elementos o introducir nuevas características. Estas prácticas son consideradas por los autores como una nueva forma de revisionismo histórico. En cuanto al proceso de ambientación de un videojuego en una determinada etapa del pasado, Salvati y Bullinger han acuñado el término selective authenticity, el cual:

may be understood as a form of narrative license, in which an interactive experience of the past blends historical representation with generic conventions and audience expectations... ${ }^{16}$.

Y arroja luz sobre “...how game designers draw upon a chain of signifiers assembled from historical texts, artefacts and popular representations " ${ }^{17}$. Esta idea, al tener en cuenta los tropos de otros medios que influencian al videojuego, se torna de gran utilidad a la hora de analizar las estrategias de autenticidad inherentes a todo producto de consumo.

La sección cuarta se centra en el análisis de elementos particulares que contribuyen a acentuar la sensación de autenticidad de determinados videojuegos históricos, como por ejemplo la arquitectura renacentista en Assassin's Creed II o los intentos de adaptar los controles de un aeroplano de la Primera Guerra Mundial en simulaciones de combates aéreos. Finalmente, los artículos agrupados en la quinta parte versan sobre videojuegos de ambientación fantástica o futurista que, sin embargo, son capaces de transmitir mensajes sobre nuestro pasado. Por ejemplo, mediante el contrafactual de una Norteamérica arrasada por el conflicto nuclear, cuyos inhóspitos parajes nos dan pistas sobre los derroteros por los que la tecnología y la cultura estadounidenses de la década de 1950 podrían haber transitado, a la vez que nos ofrecen la posibilidad de revivir procesos históricos reactualizados en un contexto similar, aunque diferente.

Las conclusiones a las que los editores del volumen llegan son especialmente in-

16 Salvati, Andrew J. y Bullinger, Jonathan M.: "Selective Authenticity and the Playable Past", en Elliott, Andrew B. R. y Kapell, Matthew Wilhelm (eds.): Playing with... p. 154.

17 Salvati, Andrew J. y Bullinger, Jonathan M.: "Selective Authenticity...", p. 154. 
teresantes. En primer lugar, van un paso más allá de Uricchio y consideran que todo videojuego histórico, independiente del tipo de simulación del pasado que realice (ya sea específica o no específica) está influenciado por una ideología determinada. Es más, este tipo de productos de consumo no tienen que ver con la historia como la academia la entiende, sino con una idea del pasado que confunde el acontecimiento con el mito, el proceso con la tradición. En este sentido, los editores siguen a autores como Bruce Lincoln y William G. Doty, quienes consideran que el mito es la ideología hecha narrativa y que estos mitos “...determine and shape ideals and goals for both individual and society"18. Elliott y Kapell no atribuyen estas características únicamente al juego digital, sino a toda forma de hacer historia. Visto de esta manera, el formato videolúdico es una forma más de darle sentido al pasado, una forma cuya idiosincrasia ha de ser comprendida por sus críticos y estudiosos.

Por otro lado, Playing with the Past explora las lógicas por las cuales estos mundos ludoficcionales aparentan ser reproducciones de entornos pretéritos. Esto ha llevado a los editores a diferenciar los términos accuracy y authenticity ${ }^{19}$. Debido a su naturaleza, los videojuegos históricos no pueden ser veraces, ya que las necesidades del medio impiden que el pasado aparezca tal y como sucedió (o como creemos que sucedió). De esta manera, la única forma de dar sentido al pasado es mediante la verosimilitud, es decir, transmitiendo una sensación histórica que es percibida por el público como la correcta. En palabras de Elliott:

From this perspective the historical significance of the digital games considered here are not - and should not be- about their historical accuracy. They are, instead, to be viewed as attempts to approach historical authenticity. Or, more directly, it is not and cannot be about 'getting the historical facts correct', but is about getting the experience and expectations of the past 'right' ${ }^{20}$.

Esta lógica convierte conceptos como selective authenticity en herramientas de extrema utilidad a la hora de analizar la construcción de discursos históricos en juegos digitales, pues permiten centrar la atención en qué elementos e ideas son considerados clave en la concepción del pasado por desarrolladores y consumidores ${ }^{21}$. Además, conecta con la noción de la historia-mito, pues muchos de los fragmentos constitutivos de las meta-narrativas históricas están moldeados por ideologías determinadas.

Siguiendo la estela de Playing with the Past, un año después se publicó una obra de similares características titulada Early Modernity and Video Games. También un esfuerzo colectivo, el libro se encuentra dividido en dos secciones: una teórica y otra práctica. Los primeros nueve capítulos utilizan como ejemplo determinados videojuegos para sacar a la luz distintas herramientas analíticas con las que elaborar una

18 Doty, William G., cited in Kapell, Matthew Wilhelm y Elliott, Andrew B. R.: "Conclusion(s): Playing at True Myths, Engaging with Authentic Histories”, en Elliott, Andrew B. R. y Kapell, Matthew Wilhelm (eds.): Playing with..., p. 362 .

19 Hemos decidido traducir estos conceptos como "veracidad" y "verosimilitud", a falta de una traducción oficial de las obras que incluyen dicha terminología.

20 Elliott, Andrew B. R. y Kapell, Matthew Wilhelm (eds.): Playing with ..., p. 361.

21 A este respecto, merece la pena señalar el trabajo de Copplerstone, Tara: "But that's not accurate: the differing perceptions of accuracy in cultural-heritage videogames between creators, consumers and critics", Rethinking History. The Journal of Theory and Practice: special issue Challenge the Past (2016), pp. 1-24. 
metodología de estudio coherente. Entre ellos destaca el de Rolf Nohr, pues utiliza el andamiaje teórico tanto de la teoría de medios como del análisis crítico del discurso para revelar los procesos mediante los cuales una realidad (en este caso, pretérita) es mediada y transformada en un interdiscurso característico, a caballo entre uno especializado y otro popular ${ }^{22}$. Los siguientes cinco capítulos son estudios de caso, la mayoría de ellos de la franquicia Assassin's Creed, y buenos ejemplos de cómo realizar el análisis de un juego de contenido histórico.

Lo realmente relevante de Early Modernity and Video Games es el esfuerzo que los editores han realizado al recoger las aportaciones de los autores y transformarlas en una propuesta de trabajo metodológica. Aunque limitada, la Historian's GameCAM proporciona al investigador un marco mediante el cual analizar las representaciones tanto estáticas como dinámicas de la historia en un videojuego. El procedimiento propuesto consta de tres pasos: C(onstrucción), A(utentificación) y M(ediación). El primero consiste en determinar la forma en la que el videojuego está construido y especificar la función que los elementos históricos cumplen en ella. Seguidamente, el apartado de Autentificación implica indagar en las estrategias de autentificación histórica del videojuego y cómo cada elemento contribuye a reforzarla, además del grado de importancia que en ellas juega. Finalmente, el paso $\mathrm{M}$ se centra en investigar cómo los elementos históricos contribuyen a que el producto sea identificado como un juego, además de las limitaciones impuestas sobre éstos por la naturaleza del medio ${ }^{23}$. A pesar del estado incompleto de esta propuesta, su aplicación junto con otros marcos metodológicos puede resultar muy beneficiosa.

Estos esfuerzos paulatinos y parciales por crear un marco teórico-metodológico propio para los historical game studies parecen haber alcanzado un nuevo estadio con la publicación en 2016 de Digital Games as History. Este libro surge como respuesta a tres grandes cuestiones: cómo aproximarse al estudio del videojuego histórico desde la perspectiva académica, cómo representa este medio el pasado y qué oportunidades ofrece al usuario para relacionarse activamente con la historia, tanto en forma de discurso como de práctica. En primer lugar, sigue la estela de Uricchio al identificar dos tipos de simulaciones enfrentadas a modo de extremos. Paralelamente, asocia cada uno de estos dos tipos a epistemologías históricas, que extrae de Alun Munslow ${ }^{24}$. De esta manera, para Chapman existen, por un lado, las simulaciones realistas $\mathrm{y}$, por otro, las simulaciones conceptuales. Las primeras son epistemológicamente similares a la historia reconstruccionista, ya que tienden a confundir el pasado con la historia, prestan especial atención al detalle y consideran su discurso histórico particular como el único posible. En cambio, las simulaciones conceptuales presentan el pasado de una forma mucho más abstracta, a la vez que ofrecen al jugador una serie de herramientas asociadas a teorías y conceptualizaciones particulares de la historia que le permiten desenvolverse en los espacios de juego. Estos productos, que sitúan al jugador al nivel cognitivo del historiador (en

22 Nohr, Rolf: "The Game is a Medium, the Game is a Message", en Winnerling, Tobias y Kerschbaumer, Florian (eds.): Early Modernity and Video Games, Newcastle upon Thyne, Cambridge Scholars Publishing, 2014, pp. $2-23$.

23 Winnerling, Tobias y Kerschbaumer, Florian: "The Devil is in the Details: Why Video Game Analysis is Such a Hard Task for Historians, and how we nevertheless try", en Winnerling, Tobias y Kerschbaumer, Florian (eds.): Early Modernity..., pp. ix-xx.

24 Munslow, Alun: Deconstructing History, Oxon, Routledge, 1997; Munslow, Alun: The New History, Harlow, Pearson, 2003. 
el sentido de que es capaz de navegar grandes distancias espacio-temporales), están próximos a epistemologías construccionistas. Como dato importante, Chapman considera que las aesthetics of historical description ${ }^{25}$ centrales difieren dependiendo del tipo de videojuego: en el caso de las simulaciones realistas predominan los elementos audiovisuales, mientras que en las conceptuales las reglas son las que adquieren mayor importancia.

Una vez establecidas dichas diferenciaciones (que, recordemos, son extremos opuestos de los discursos que los videojuegos pueden enunciar sobre el pasado), Chapman ofrece un marco metodológico para su estudio compuesto por una serie de categorías analíticas clave. En primer lugar, encontramos el tiempo y el espacio. A pesar de ser categorías separadas, su íntima relación hace que en la mayoría de los casos sean estudiadas paralelamente. En lo que respecta al espacio, existen dos tipos: los "jardines narrativos" y los "lienzos narrativos". El modelo de jardín sigue una estructura lineal, en la que el jugador ha de superar obstáculos desde una línea de salida hasta alcanzar una meta. A pesar del número de desvíos que el usuario pueda tomar, éstos convergen en un lugar concreto, lo que supondrá un punto y seguido (o punto y final) a la narración. En cambio, el modelo de lienzo tiende a no tener principio o fin definidos y el espacio se entiende como un recurso sobre el cual edificar discursos históricos. De esta manera, el jugador va colocando elementos históricos (edificios, unidades, etcétera) sobre este lienzo, generando su propio relato. En cuanto al tiempo, Chapman detecta tres tipos: el tiempo real, el tiempo ficcional (es decir, el diegético) y el tiempo histórico. Mientras que, en los juegos realistas, que tienden a utilizar el espacio como jardín narrativo, la relación de estos tiempos es simétrica (1:1:1), en las simulaciones conceptuales con lienzos narrativos esta proporción suele alterarse para adecuarse a la perspectiva del historiador: el tiempo real se presta a transcurrir mucho más rápido que el diegético, además de que el histórico se acelera, pudiendo transcurrir décadas en cuestión de minutos.

Las siguientes categorías tienen que ver con modelos narrativos, tanto los característicos de los videojuegos como aquellos asociados a la escritura de la historia. Por tanto, vuelven a ser dos esferas convergentes. Para el correcto análisis ludonarrativo ${ }^{26}$ de un juego digital, Chapman propone la deconstrucción del sistema en lexia, framing controls y framing objectives. Todo juego propone unos objetivos (framing objectives), cuyo alcance o inaccesibilidad determinará la victoria o derrota del usuario. Para alcanzar estos objetivos, el jugador ha de organizar de manera específica los distintos lexia presentes en el espacio de juego (todos aquellos elementos con los que se pueda interactuar, por ejemplo, un catalejo, una mina de plata o una carabela) ayudándose de los controles que el desarrollador le ha concedido (framing controls $)^{27}$. La forma en la que un videojuego presenta sus lexia, framing controls y

25 Este concepto alude a las estrategias que los productos videolúdicos siguen a la hora de otorgarle significados al pasado, así como a los elementos que componen dichas estrategias (sonidos, banda sonora musical, representaciones visuales, reglas de juego, descripciones enciclopédicas, etcétera).

26 Este concepto fue acuñado por Marie-Laurie Ryan como forma de conciliar las interpretaciones narratológicas del videojuego con las concepciones de los ludólogos. En resumen, la ludonarrativa es considerada la forma característica de narración de un videojuego, pues la ficción se desarrolla a través de una serie de reglas que gobiernan el sistema. Para más información, ver Planells de la Maza, Antonio J.: Videojuegos..., pp. 83-89.

27 Con el fin de ilustrar la teoría, pongamos que en un hipotético juego de combate de la Segunda Guerra Mundial el objetivo es tomar el ayuntamiento de una ciudad de Normandía (framing objective). Para ello, el jugador ha de abatir a los soldados enemigos con un repertorio armamentístico compuesto por una pistola, un rifle y granadas (tanto los soldados como el armamento son lexia, que ha de organizar de manera que los combatientes 
framing objectives determinará qué tipo de narrativa poseerá. Aquellos con framing goals explícitos y constantes, una gran cantidad de framing narrative (momentos en los que el jugador pierde el control) y cuya ludonarrativa no altere el argumento general del juego se adscriben a narrativas deterministas. En cambio, Chapman considera próximas a narrativas ontológicas aquellas simulaciones en las que el control del usuario es constante, los framing goals son escasos e implícitos y el discurso está fuertemente condicionado por el desarrollo de la ludonarrativa. A medio camino se sitúan las narrativas abiertas, que presentan un balance entre las narrativas de creación características de los modelos ontológicos y las de descubrimiento propias de las deterministas. Por lo general, las simulaciones realistas tienden a ser deterministas, mientras que las conceptuales son de naturaleza ontológica.

A la hora de aplicar estas categorías narrativas a la representación del pasado, salen a la luz procesos destacables. Por un lado, hay que tener en cuenta que el videojuego histórico, como cualquier forma de discurso histórico, es mimético: utiliza elementos referenciales al pasado para construir significados. La potencialidad narrativa de los modelos anteriormente descritos permite, por tanto, la emergencia de una explicación u otra del pasado. Mientras que los juegos realistas y deterministas suelen transmitir interpretaciones fijas sobre el pasado, aquellos ontológicos y conceptuales tienden a asemejarse a debates teóricos sobre el pasado. Por otro lado, las formas y contenidos presentes en la narrativa histórica videolúdica han de adaptarse a las necesidades del medio. Esto hace que algunos elementos históricos sean excluidos de la narración, al considerarse que impiden el objetivo último de la forma lúdica: la competición y el entretenimiento. A pesar de ello, las tensiones entre este formato y los modos tradicionales de presentación del discurso histórico hacen que, en ocasiones, se produzcan disonancias en las narrativas lúdico-históricas, esto es, una contradicción entre la historia, entendida como relato, y el juego. El estudio de estas disonancias se torna conveniente, pues transmite no sólo las limitaciones del videojuego como forma histórica, sino que nos puede hacer reflexionar sobre nuestra forma de entender el pasado y relacionarnos con él.

Finalmente, la última categoría de análisis está compuesta por las anteriormente mencionadas affordances. En palabras de Chapman:

Games, as particularly interactive media, are especially concerned with what the relationships of the past afforded (what could or could not be done) rather than simply what things or events were, at least in their gameplay (...) Representations of these historical affordances, in turn, must offer players new gameplay affordances, which yet must generally in some way say something about the claimed nature of the original relationship ${ }^{28}$.

De esta manera, los juegos históricos pueden comportarse de tres maneras distintas dependiendo de las affordances que representen y permitan: como museos, como formas de recreación histórica y como herramientas de exploración de contrafactuales. Los primeros funcionan como espacios digitales de historia viva, de forma

sean eliminados), ayudándose de su habilidad para correr, agacharse, apuntar, disparar, recargar... (framing controls).

28 Chapman, Adam: Digital Games as History: How Videogames Represent the Past and Offer Access to Historical Practice, Oxon/New York, Routledge, 2016, p. 174. 
similar a los centros de interpretación, en los que el jugador puede recorrer enclaves pretéritos y observar relaciones y prácticas históricas, pudiendo incluso convertirse en participante de las mismas. Por otro lado, los juegos de recreación histórica poseen una serie de características que los diferencian de las prácticas de recreación tradicionales. La ausencia de interacción corporal con el videojuego hace que los retos a los que se enfrente el jugador sean fundamentalmente visuales, consistentes en detectar affordances históricas en lugar de realizarlas: por ejemplo, identificar la trayectoria exitosa de un disparo en lugar de levantar un pesado rifle, apuntar y apretar el gatillo. En lo que respecta al potencial contrafactual de los juegos, las affordances que permiten son similares a las del historiador profesional:

Players are offered the possibility of becoming player-historians, experiencing freedom to engage in historical practices and yet doing so in a structured story space in which much of the groundwork is already complete ${ }^{29}$.

A pesar de este potencial, Chapman advierte de las limitaciones que los videojuegos imponen a sus jugadores y que afectan de forma similar al historiador, aunque en un grado distinto:

However, these games also feature significant tensions in their tendency for counterfactual history, their intrinsically structuralist approach to history and their enfranchisement of players into the construction of historical narrative. These tensions connect with a number of existing historiographical, methodological ideological and epistemological debates ${ }^{30}$.

Finalmente, no queremos clausurar la presente nota sin hacer referencia al estado de la cuestión en España. Ésta se encuentra en estado embrionario, puesto que el único impulso relevante ha sido la publicación de la colección "Historia y Videojuegos" como resultado del proyecto de investigación homónimo realizado en el seno del Centro de Estudios Medievales de la Universidad de Murcia (referencia: HAR2011$22548)^{31}$.

En resumen, el repaso a los principales hitos bibliográficos de la naciente disciplina de los historical game studies nos ha permitido, en primer lugar, situar esta línea de investigación dentro de las últimas tendencias en las Humanidades, en especial en relación con el postestructuralismo. Por otro lado, se ha puesto de manifiesto la capacidad del videojuego para articular discursos históricos coherentes y que dotan al pasado de un significado válido. De esta manera, la forma lúdica queda elevada al mismo nivel que otros formatos como el escrito (considerado hasta muy recien-

29 Chapman, Adam: Digital Games..., pp. 232-233.

30 Chapman, Adam: Digital Games..., p. 233.

31 La colección cuenta con tres volúmenes: San Nicolás Romera, César y Nicolás Ojeda, Miguel Ángel (comps.): Videojuegos y sociedad digital: nuevas realidades de estudio para la percepción del pasado histórico. (Colección Historia y Videojuegos, volumen 1), Mar del Plata, Universidad Nacional de Mar del Plata, 2015; Jiménez Alcázar, Juan Francisco; Mugueta Moreno, Íñigo y Rodríguez, Gerardo Fabián (eds.): Historia y Videojuegos: el impacto de los nuevos medios de ocio sobre conocimiento histórico (Colección Historia y Videojuegos, volumen 2), Murcia, Centro de Estudios Medievales de la Universidad de Murcia/Editorial Compobell/IGN España, 2016; Jiménez Alcázar, Juan Francisco: De la Edad de los Imperios a la Guerra Total: Medievo y Videojuegos (Colección Historia y Videojuegos, volumen 3), Murcia, Centro de Estudios Medievales de la Universidad de Murcia/Editorial Compobell/IGN España, 2016. 
temente como el único realmente válido), el fílmico o el oral. En tercer lugar, se han introducido una serie de conceptos de máxima utilidad para el análisis de productos de entretenimiento históricos particulares. Ideas como la verosimilitud o las affordances se convierten en lupas mediante las cuales observar el fenómeno de la historia lúdica, ya que revelan las estrategias y tendencias narrativas características del medio. Finalmente, se han puesto al alcance de la comunidad científica hispanohablante los principales esfuerzos teóricos realizados por la academia anglosajona por dotar a los videojuegos históricos de un estatus epistemológico, además de proporcionar los marcos metodológicos requeridos para el análisis sistemático de estas obras culturales. 